\title{
Evaluation of the clinical staging of esophageal cancer by using diffusion-weighted imaging
}

\author{
TOMOYOSHI AOYAGI ${ }^{1}$, KIYOHIKO SHUTO ${ }^{1}$, SHINICHI OKAZUMI ${ }^{1}$, HIDEAKI SHIMADA ${ }^{1}$, \\ YOSHIHIRO NABEYA ${ }^{1}$, TOSHIKI KAZAMA ${ }^{2}$ and HISAHIRO MATSUBARA ${ }^{1}$
}

Departments of ${ }^{1}$ Frontier Surgery and ${ }^{2}$ Radiology, Graduate School of Medicine, Chiba University, Chiba 260-8670, Japan

Received April 6, 2010; Accepted June 10, 2010

DOI: $10.3892 /$ etm.2010.112

\begin{abstract}
The purpose of this study was to assess the effectiveness of the apparent diffusion coefficient (ADC) using diffusion-weighted imaging (DWI) for the clinical staging of esophageal cancer. The ADC of the tumors was compared to the standardized uptake value (SUV) of ${ }^{18} \mathrm{~F}$-fluorodeoxyglucose-positron emission tomography (FDG-PET). A total of 123 consecutive patients with esophageal squamous cell cancer were enrolled. DW images were obtained using a $1.5 \mathrm{~T}$ whole-body scanner equipped with a phased-array body coil. A single shot spin-echo type of echo-planar sequence that provided diffusion weighting in the direction of the slice selection was used to obtain DW images. There was a negative correlation between the ADC value of the tumors and tumor diameter. A negative correlation was also found between the ADC value/SUV of the FDG-PET and serum levels of the tumor markers squamous cell carcinoma antigen and CYFRA. The ADC value was significantly correlated with clinical $\mathrm{T}$ and $\mathrm{N}$ stages. The ADC values of advanced-stage tumors were significantly lower than those of early-stage tumors. The ADC value of esophageal cancer decreased as the clinical stage advanced, and was correlated with the clinical findings. This non-invasive modality may be a valid clinical imaging method for the staging of esophageal cancer.
\end{abstract}

\section{Introduction}

Diffusion-weighted imaging (DWI) of the brain is routinely used for the early detection and characterization of cerebral stroke (1). Due to its exquisite sensitivity to motion, the execution of DWI of the abdomen has until recently faced numerous obstacles. With the advent of the echo-planar magnetic resonance imaging (MRI) technique (2-5), diffusion-weighted MRI of the abdomen over a short time interval has become

Correspondence to: Dr Tomoyoshi Aoyagi, Department of Frontier Surgery, Graduate School of Medicine, Chiba University, 1-8-1 Inohana, Chuo-ku, Chiba-shi, Chiba 260-8670, Japan

E-mail: aotomo@d2.dion.ne.jp

Key words: apparent diffusion coefficient, diffusion-weighted image, esophageal cancer, magnetic resonance imaging possible, minimizing the effect of physiologic motion such as respiration and cardiac movement. MRI is commonly used in cancer therapy to determine the involvement of macroscopic structures for tumor detection and staging. Techniques for evaluating the characteristics of tumors, such as endoscopic ultrasound, are usually invasive, and are therefore not included in routine examinations. A non-invasive method that provides necessary information, such as location and tumor characteristics, may involve the measurement of water proton mobility using DWI.

Patients with esophageal cancer have a poor prognosis, since most tumors are at an advanced stage at the time of diagnosis, which is usually the time of onset of symptoms. Resection is the only curative method for esophageal cancer, but is limited to early-stage disease. Therefore, early diagnosis and accurate staging are crucial to determine the possibility of surgery. Accurate tumor staging is also critical for the selection of therapy and the prediction of prognosis.

Various tissues have unique diffusion characteristics determined using the apparent diffusion coefficient (ADC). Tumor structure is known to change as a cancer grows and advances in stage. Folpe et al reported that this change in tumor structure may be reflected in clinical imaging (6). However, the relationship between ADC values and clinical findings or the stages of esophageal tumors is unclear. In this study, we assessed the progression of esophageal squamous cell carcinoma using DWI by monitoring changes in tumor diffusion, which reflect changes in interstitial water volume and cellular permeability.

The purpose of this study was to assess the correlation between clinical status (tumor diameter, serum tumor marker values and TNM staging) and ADC values calculated by DWI in cases of esophageal squamous cell carcinoma (ESCC). The $\mathrm{ADC}$ value of the tumors was compared to the standardized uptake value (SUV) of ${ }^{18} \mathrm{~F}$-fluorodeoxyglucose-positron emission tomography (FDG-PET).

\section{Materials and methods}

Patient population. A total of 123 patients (107 men, 16 women; mean age 65.4 \pm 7.7 years; range 44-82 years), admitted to our institution between January 2006 and March 2008 were enrolled in the study. All patients had primary SCC of the thoracic esophagus, histopathologically diagnosed 
using endoscopic biopsy or surgical resection specimens. Of the patients, 31 patients underwent curative esophagectomy, 84 were treated by radiotherapy, chemotherapy or both, and 8 were not treated. A detailed examination was performed by esophagoscopy, endoscopic ultrasound, multi-detector row computed tomography (MDCT), esophagography and FDG-PET. Endoscopic ultrasound and esophagography are superior for the diagnosis of early-stage esophageal cancer, while MDCT is superior for advanced-stage cancer. The clinical stages of the 123 patients were determined according to the International TNM classification (7): T1, 21 patients; T2, 10; T3, 47; T4, 45; N0, 28; N1, 95; and stage I, 18; stage II, 16; stage III, 52; and stage IV, 37. Serum levels of the tumor markers SCC antigen, carcinoembryonic antigen (CEA) and CYFRA were also determined.

Informed consent according to the Declaration of Helsinki was obtained from all patients prior to treatment. The study was approved and carried out according to the guidelines for clinical research of our institutional review boards.

Diffusion-weighted imaging. MRI was performed using a 1.5-Tesla body scanner equipped with a phased array body coil (Achiva 1.5T Nova Dual; Philips Medical, Best and Heeren, The Netherlands). Images were obtained in the following sequences: a single-shot spin-echo type of echo-planar sequence was used to obtain DWI; fat signals were suppressed using short-tau inversion recovery (8). The b-values corresponding to diffusion-sensitizing gradients were $b=0$ and $1,000 \mathrm{sec} / \mathrm{mm}^{2}$. Sequential sampling of the $\mathrm{k}$-space was used with an effective echo time and an acquisition matrix of $128 \times 128$, which was interpolated to $256 \times 256$ during image calculation. Forty consecutive slices from the clavicle to the diaphragm were acquired to cover the entire tumor with a 400-mm field of view, a 4-mm slice thickness and a 1-mm slice gap. DWI was performed in $7 \mathrm{~min}$ with free breathing scanning. Before DWI, $\mathrm{T}_{2}$-weighted images were obtained in the transverse plane. $\mathrm{T}_{2}$-weighted fast spin-echo images were obtained with the following parameters: TR/TE 3000/75, train length of 15; acquisition of four signals, 192×256 matrices, $32-\mathrm{cm}$ field of view and $8-\mathrm{mm}$ section thickness. The number of excitations was six to average the motion artifacts. There were no differences in image quality depending on the location. The patients that did not receive pre-treatment underwent examination 1 week prior to surgery.

To minimize esophageal movement, a minimum of $6 \mathrm{~h}$ of fasting with no restrictions on drinking water was required before the examination. Immediately before the examination, $20 \mathrm{mg}$ of N-butyl scopolamine bromide was administered by intramuscular injection.

Image analysis. The ADC map of each slice was generated on a pixel-by-pixel basis from the signal intensity of the DWI images and the corresponding b-values based on the equation: $\mathrm{ADC}=\log \mathrm{S}(0)-\log \mathrm{S}(1,000)$.

A natural logarithm was used in which $S(b)$ represented the signal intensity with the diffusion gradient, $S(0)$ the signal intensity without the diffusion gradient and $\mathrm{b}$ the gradient factor (in sec/ $\mathrm{mm}^{2}$ ) of the used pulse sequence as a measure of strength of the diffusion gradient. Regions of interest were established in each lesion on the mapping images. ADC maps
A

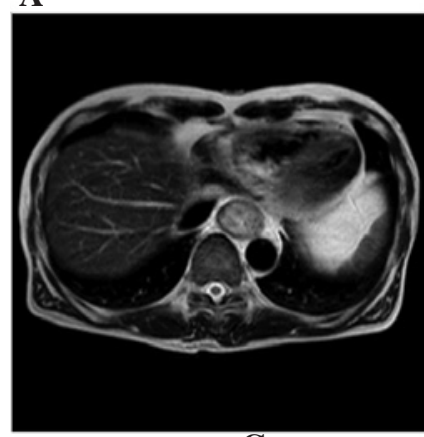

B

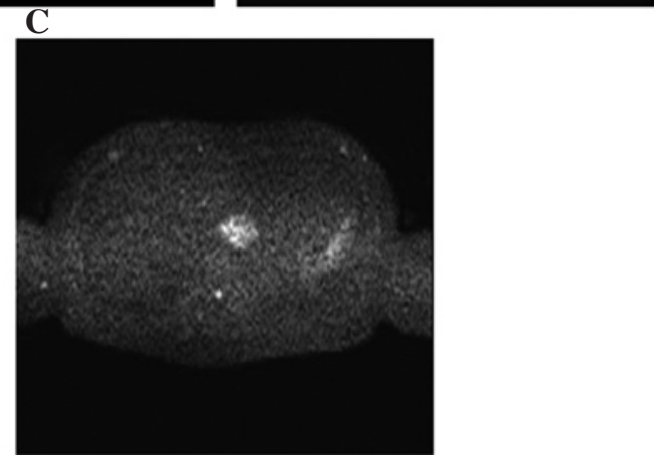

Figure 1. Transverse MR images in a 60-year-old man with esophageal squamous cell carcinoma. (A) Representative $\mathrm{T}_{2}$-weighted image of an esophageal tumor. The tumor tissue shows high intensity. (B) Representative DWI of an esophageal tumor. The image was captured at the same level as A. The tumor tissue shows high intensity (white-black revised). (C) The ADC map of each slice was generated on a pixel-by-pixel basis from the signal intensity of the DWI images and the corresponding b-values. The image was captured in the same level as A and B. The ADC value was $1.020 \times 10^{-3} \mathrm{~mm}^{2} / \mathrm{sec}$. The $\mathrm{ADC}$ of the tumor tissue was lower than that of the normal esophageal tissue.

were calculated and transferred to a remote $\mathrm{PC}$ workstation (Aze Virtual Place Advanced Plus, Japan).

The regions of interest were drawn on DWI by two investigators experienced in MRI for 10 and 19 years, respectively. Tumor locations were defined based on MDCT, esophagography, esophagoscopy and MRI ( $\mathrm{T}_{2} \mathrm{WI}$ and $\left.\mathrm{DWI}\right)$. The representative area of the tumor was determined according to the irregularity of the wall layer, tumor density and difference in intensity compared to the surrounding area. Due to excellent fat suppression with background body-signal suppression, fat around the esophagus had a very low signal on DWI. An ADC value was obtained from 10 regions of interest from different axial section levels of the same lesion. All regions of interest were placed within the lesions and traced on the ADC maps. The ADC values then were measured using the ADC maps. The image quality of the DWI and the acquired ADC maps were sufficient to identify the tumor region in all patients. The ADC values were compared to tumor diameter, the serum tumor marker value, the SUV of FDG-PET and TNM stage.

Statistical analysis. Spearman-rank correlations were used to assess the relationship between ADC values and tumor diameter, serum tumor markers and SUV of the FDG-PET. We measured the tumor diameter using esophagography. The Kruskal-Wallis test was used for overall comparison, and the Mann-Whitney U test was used for comparison between two groups (for example, between the ADC values of cancer tissue and normal esophagus) and Dunn's multiple comparison test. 


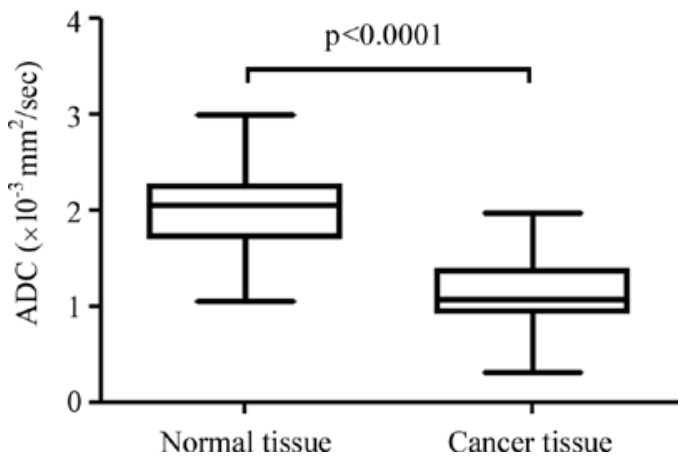

Figure 2. Comparison of the mean ADC values of normal esophageal and cancer tissue. The ADC values (vertical axis) are plotted against the normal and cancer tissues. The median value is the horizontal line within a box. Error bars indicate the 5 and 95 percentiles $(\mathrm{P}<0.0001)$.

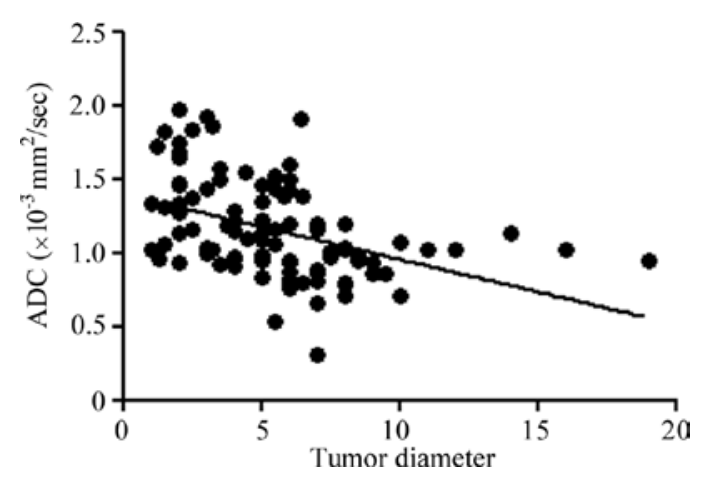

Figure 3. Correlation between ADC and tumor diameter. A significant negative linear correlation was found between ADC and tumor diameter $(\mathrm{P}<0.0001)$.

The cutoff value was set according to receiver operating characteristic (ROC) analysis. The differences were considered significant when P-values were $<0.05$. All statistical analyses were performed using GraphPad Prism software (La Jolla, CA, USA). The values are expressed as the mean \pm standard deviation (SD).

\section{Results}

Clinical characteristics of tumors and ADC values. Representative $\mathrm{T}_{2^{-}}$and diffusion-weighted images are shown in Fig. 1A and $\mathrm{B}$; a representative ADC map is shown in Fig. 1C.

The ADC values of the cancer tissue were significantly lower than those of the normal esophagus $\left(1.145 \pm 0.321 \times 10^{-3}\right.$ vs. $2.001 \pm 0.385 \times 10^{-3} \mathrm{~mm}^{2} / \mathrm{sec}$, respectively; $\mathrm{P}<0.0001$ ) (Fig. 2). ROC analysis was performed to establish a statistical standard to diagnose the cancer or normal tissue. According to ROC analysis, the cutoff value was set at $1.5 \times 10^{-3} \mathrm{~mm}^{2} / \mathrm{sec}$, the sensitivity was $92 \%$, the specificity $86 \%$ and the accuracy $89 \%$.

The ADC values were compared to the clinical tumor characteristics, tumor diameter, serum levels of tumor markers and the SUV of FDG-PET. The ADC value decreased as the tumor advanced in stage. A negative correlation was found between the ADC values of the tumors and tumor diameter $\left(\mathrm{R}^{2}=0.1859, \mathrm{P}<0.0001\right.$; Fig. 3).
A

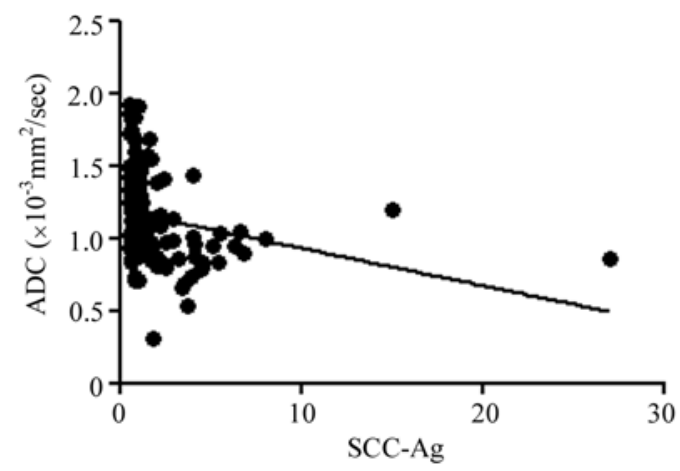

B

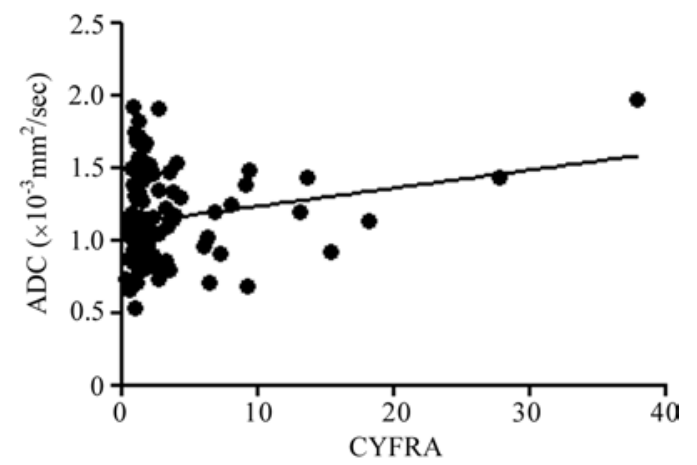

Figure 4. Correlation between ADC and tumor markers. A significant negative linear correlation was noted between ADC and tumor markers, serum squamous cell antigen and CYFRA $(\mathrm{P}=0.0074$ and $=0.0341$, respectively $)$.

Regarding the tumor markers, a negative correlation was also noted between the ADC of the tumors and SCC antigen $\left(\mathrm{R}^{2}=0.0646, \mathrm{P}=0.0074\right)$ and CYFRA $\left(\mathrm{R}^{2}=0.0445, \mathrm{P}=0.0341\right)$. However, a significant correlation with serum CEA was not observed $\left(\mathrm{R}^{2}=0.0133\right.$ and $\mathrm{P}=0.6990$, respectively) (Fig. 4).

The SUV of FDG-PET showed a negative correlation with ADC $\left(\mathrm{R}^{2}=0.1581, \mathrm{P}<0.0001\right.$; Fig. 5). The SUV of FDG-PET presented maximum values of the tumors.

Clinical TNM staging and ADC value. The ADC values between the cancer samples and different clinical TNM stages were compared. The ADC values of clinical (c)T1, 2,3 and 4 were $1.443 \pm 0.247,1.439 \pm 0.375,1.074 \pm 0.246$ and $1.016 \pm 0.287 \times 10^{-3} \mathrm{~mm}^{2} / \mathrm{sec}$, respectively. The ADC of cT1 tumors was significantly higher than that of cT3 $(\mathrm{P}<0.05)$ and cT4 $(\mathrm{P}<0.05)$ tumors, while the ADC value of cT2 tumors was higher than cT3 $(\mathrm{P}<0.05)$ and $\mathrm{T} 4(\mathrm{P}<0.05)$ tumors (Table I). Additionally, the ADC of tumors of $\mathrm{cNO}$ was higher than that of $\mathrm{cN} 1$ tumors $\left(1.390 \pm 0.293\right.$ and $1.073 \pm 0.294 \times 10^{-3} \mathrm{~mm}^{2} / \mathrm{sec}$, respectively; $\mathrm{P}<0.0001$ ) (Table I). The ADC values of cStages I, II, III and IV were $1.443 \pm 0.328,1.347 \pm 0.333,1.062 \pm 0.253$ and $1.030 \pm 0.307 \times 10^{-3} \mathrm{~mm}^{2} / \mathrm{sec}$, respectively. ADC values of advanced-stage tumors were significantly lower than those early-stage tumors, and a significant difference between cStage I and III $(\mathrm{P}<0.05)$, I and IV $(\mathrm{P}<0.05)$, II and III $(\mathrm{P}<0.05)$ and II and IV $(\mathrm{P}<0.05)$ was observed (Table I).

\section{Discussion}

By applying a pair of strong magnetic field gradient pulses within the imaging sequence, imaging is sensitized to random spin displacements of diffusing free water molecules $(1,9)$. 


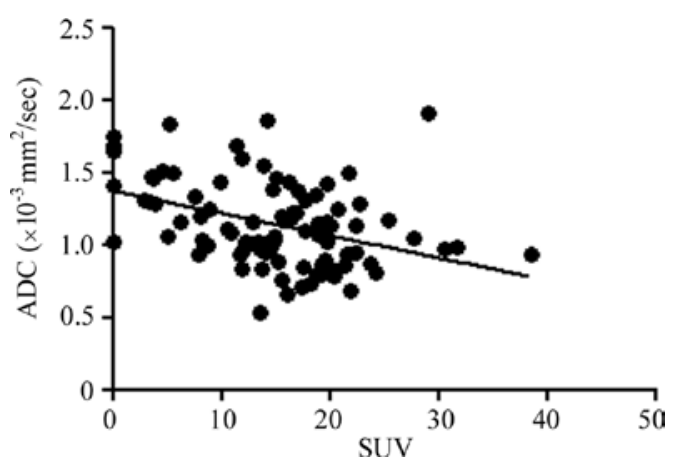

Figure 5. Correlation between ADC and SUV of FDG-PET. A significant negative linear correlation was found between ADC and SUV of FDG-PET $(\mathrm{P}<0.001)$.

ADC values are calculated from DWI measurements, and depend on the impediment of the free diffusion of water molecules in a single voxel due to restricting barriers, such as membranes, macromolecules and fibers inside different tissue compartments. Therefore, DWI has been suggested as a tool to distinguish different tissue compartments and to detect changes in cellular tissue structure, which are used to monitor the effects of radiation in tumor tissue. Various tissues have unique diffusion characteristics as measured using ADC. We did not perform a perfusion study of the tumors since, according to De Vries et al, there was no apparent association between changes in the ADC values and perfusion data obtained for the same patients (10). The ADC value seemed to represent mainly changes in water molecule mobility.

DWI and ADC mapping in the radiologic diagnosis of neoplasms was initially suggested to be useful for the analysis of the central nervous system, and the difference in tissue diffusion was thought to depend on the difference in the structure of the tumor tissue (11-16). There have been few reports of DWI applied to the gastrointestinal tract. Some reports have been published on DWI in colorectal cancer (17-19), but DWI in esophageal cancer has only been reported once (20). The reason for this may be that the esophagus is surrounded by air in the lung, and the MR images are greatly distorted since the uniformity of the magnetic field is disturbed. Recent improvements and the introduction of DWI with background body signal suppression allows for the acquisition of satisfactory DWI of the esophagus. In this study, we confirmed that DWI was useful for detecting esophageal cancer, despite respiratory and cardiac motion.

The tissue ADC value is thought to be based mainly on independent contributions of the extracellular and intracellular tissue compartments $(21,22)$. Due to the larger volume content of relatively impermeable impeding barriers, such as cell and matrix fibers, lower ADC values have been observed in the intracellular compartment (23). Thus, changes in ADC value are thought to reflect changes in the extracellular volume (ECV)/intracellular volume (ICV) ratio; increased ICV ultimately leads to a lower ADC value. The ADC value is a reference for restrictions in molecular diffusion caused by structures, allowing inferences to be made about the microstructure of the cellular environment. In other words, the ADC value reflects cellularity and cell density. The minimum ADC value of high-grade gliomas was reported to be lower than
Table I. Clinical TNM staging and the ADC value.

\begin{tabular}{lcc}
\hline Clinical findings & ADC $\left(\times 10^{-3} \mathrm{~mm}^{2} / \mathrm{sec}\right)$ & P-value \\
\hline Tumor depth & & $<0.0001^{\mathrm{a}}$ \\
cT1 & $1.443 \pm 0.247$ & \\
cT2 & $1.439 \pm 0.375$ & \\
cT3 & $1.074 \pm 0.246$ & \\
cT4 & $1.016 \pm 0.287$ & \\
Node metastasis & & $<0.0001^{\mathrm{b}}$ \\
cN0 & $1.390 \pm 0.293$ & \\
cN1 & $1.030 \pm 0.307$ & \\
TNM stages & & \\
cT1 & $1.443 \pm 0.328$ & \\
cT2 & $1.347 \pm 0.333$ & \\
cT3 & $1.062 \pm 0.253$ & \\
cT4 & $1.030 \pm 0.307$ & \\
\hline
\end{tabular}

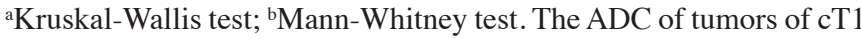
was significantly higher than that of cT3 $(\mathrm{P}<0.001)$ and cT4 tumors $(\mathrm{P}<0.0001)$; ADC of cT2 tumors was higher than that for $\mathrm{cT} 3(\mathrm{P}<0.05)$ and cT4 tumors $(\mathrm{P}<0.01)$. Also, $\mathrm{ADC}$ of tumors of $\mathrm{cN} 0$ was higher than that for tumors of $\mathrm{cN} 1\left(1.335 \pm 0.307\right.$ and $1.061 \pm 0.286 \times 10^{-3} \mathrm{~mm}^{2} / \mathrm{sec}$, respectively; $\mathrm{P}<0.001)$. ADC values of advanced-stage tumors were significantly lower than that of early-stage tumors, and a significant difference between cStage I and III $(\mathrm{P}<0.01)$, I and IV $(\mathrm{P}<0.0001)$, II and IV $(\mathrm{P}<0.001)$ and III and IV $(\mathrm{P}<0.01)$ was found.

that of low-grade gliomas (14). Published data on intracranial tumors indicate that a high ADC value may be attributable to low cellularity, while lower values are attributable to dense and high cellularity $(15,16)$.

In this study, we measured $\mathrm{ADC}$ values in viable areas of esophageal cancer, excluding necrotic areas identified by esophagoscopy and enhanced MDCT, since the viable area of the cancer tissue is what reflects the malignant potential, clinically manifested as growth in size, an increased serum tumor marker level, increased SUV and advanced TNM stage. However, visible necrotic areas were rarely found, and the microscopic necrotic areas were not excluded. The ADC values of the normal esophagus were higher than that of the cancer tissue.

We found a negative correlation between the ADC values and tumor diameter, the SUV of the FDG-PET, serum SCC antigen and CYFRA levels, and the clinical staging of ESCC. Many studies have suggested an independent association between SUV and proliferative activity or cellularity in SCC (24-27). Higher cellularity was noted in advanced ESCC, with extensive infiltration and increased lymph node involvement, and higher malignant potential. SCC-Ag concentration and SCC-Ag positivity rates in ESCC are known to be significantly elevated in patients with advanced-stage tumors. SCC-Ag concentrations and SCC-Ag positivity rates were found to be significantly elevated in tumors with increased size, tumor depth, positive lymph node metastasis and distant metastasis (28). We compared ADC values between cancers and different clinical TNM stages. The ADC values of clinical $\mathrm{T}$ and $\mathrm{N}$ stages decreased with increasing clinical stage. Advanced-stage tumors were anticipated to have high 
cellularity, thus we interpreted that the ADC values had a negative correlation among clinical stages. According to the variance of measured ADC values, 10 ROIs per lesion were measured by two investigators with extensive experience in MRI. The ADC values of one lesion per person tended to be similar. Therefore, we regarded possible image artifacts and measurement errors as minimal.

In conclusion, this study showed that the calculation of ADC values using DWI is useful for assessing the stage of ESCC, since the ADC values corresponded to clinical findings such as tumor diameter, serum tumor marker levels and clinical $\mathrm{T}$ and $\mathrm{N}$ stages. Further study of non-invasive radiological assessment is required to improve the management of malignant tumors by predicting response to therapy with optimum characterization of the tumor.

DWI enables the visualization of changes in molecular diffusion within tissues related to ESCC progression. Moreover, it is a valuable modality, as there is no exposure to radiation, the examination duration is short and the test is relatively inexpensive. There are many diagnostic imaging methods, but we often use MDCT, esophagography, esophagoscopy, endoscopic ultrasound, MRI and FDG-PET for clinical staging. MDCT, FDG-PET and esophagography are associated with exposure to radiation and contrast agents, and endoscopic ultrasound and esophagoscopy are invasive methods of diagnosis. For these reasons, this non-invasive modality may be an appropriate method for screening malignancies, and a substitute for FDG-PET. To the best of our knowledge, there are no prior reports on DWI in esophageal cancer, while only a few on rectal cancer in response to treatment can be found $(17,18)$. In conclusion, DWI may be used as a valid clinical imaging modality to evaluate the malignant potential of ESCC.

\section{References}

1. Mori S and Barker PB: Diffusion magnetic resonance imaging: its principle and applications. Anat Rec 257: 102-109, 1999.

2. Butts K, Riederer SJ, Ehman RL, Felmlee JP and Grimm RC: Echo-planar imaging of the liver with a standard MR imaging system. Radiology 189: 259-264, 1993.

3. Edelman RR, Wielopolski P and Schmitt F: Echo-planar MR imaging. Radiology 192: 600-612, 1994.

4. Muller MF, Prasad P, Siewert B, Nissenbaum MA, Raptopoulos V and Edelman RR: Abdominal diffusion mapping with use of a whole-body echo-planar system. Radiology 190: 475-478, 1994.

5. Keogan MT and Edelman RR: Technologic advances in abdominal MR imaging. Radiology 220: 310-320, 2001.

6. Folpe AL, Lyles RH, Sprouse JT, Conrad EU III and Eary JF: (F-18) fluorodeoxyglucose positron emission tomography as a predictor of pathologic grade and other prognostic variables in bone and soft tissue sarcoma. Clin Cancer Res 6: 1279-1287, 2000.

7. Sobin LH and Fleming ID: TNM Classification of Malignant Tumors, 5th edition (1997). Union Internationale Contre le Cancer and the American Joint Committee on Cancer. Cancer 80: 1803-1804, 1997.

8. Takahara T, Imai Y, Yamashita T, Yasuda S, Nasu S and van Cauteren M: Diffusion weighted whole body imaging with background body signal suppression (DWIBS): technical improvement using free breathing, stir and high resolution 3D display. Radiat Med 22: 275-282, 2004.

9. Eo S and Je T: Spin diffusion measurements: spin echoes in the presence of time-dependent field gradients. J Chem Phys 42: 288-292, 1965.

10. De Vries A, Griebel J, Kremser C, et al: Monitoring of tumor microcirculation during fractionated radiation therapy in patients with rectal carcinoma: preliminary results and implications for therapy. Radiology 217: 385-391, 2000.
11. Hayashida Y, Hirai T, Morishita S, et al: Diffusion-weighted imaging of metastatic brain tumors: comparison with histologic type and tumor cellularity. AJNR Am J Neuroradiol 27: 1419-1425, 2006.

12. Mardor Y, Pfeffer R, Spiegelmann R, et al: Early detection of response to radiation therapy in patients with brain malignancies using conventional and high b-value diffusion-weighted magnetic resonance imaging. J Clin Oncol 21: 1094-1100, 2003.

13. Asao C, Korogi Y, Kitajima M, et al: Diffusion-weighted imaging of radiation-induced brain injury for differentiation from tumor recurrence. AJNR Am J Neuroradiol 26: 1455-1460, 2005.

14. Yang D, Korogi Y, Sugahara T, et al: Cerebral gliomas: Prospective comparison of multivoxel 2D chemical-shift imaging proton MR spectroscopy, echoplanar perfusion and diffusion-weighted MRI. Neuroradiology 44: 656-666, 2002.

15. Tien RD, Felsberg GJ, Friedman H, Brown M and MacFall J: MR imaging of high-grade cerebral gliomas: value of diffusionweighted echoplanar pulse sequences. AJR Am J Roentgenol 162: 671-677, 1994.

16. Sugahara T, Korogi Y, Kochi M, et al: Usefulness of diffusionweighted MRI with echo-planar technique in the evaluation of cellularity in gliomas. J Magn Reson Imaging 9: 53-60, 1999.

17. Dzik-Jurasz A, Domenig C, George M, et al: Diffusion MRI for prediction of response of rectal cancer to chemoradiation. Lancet 360: 307-308, 2002.

18. Hein PA, Kremser C, Judmaier W, et al: Diffusion-weighted magnetic resonance imaging for monitoring diffusion changes in rectal carcinoma during combined, preoperative chemoradiation: preliminary results of a prospective study. Eur J Radiol 45: 214-222, 2003

19. Kremser C, Judmaier W, Hein P, Griebel J, Lukas P and de Vries A: Preliminary results on the influence of chemoradiation on apparent diffusion coefficients of primary rectal carcinoma measured by magnetic resonance imaging. Strahlenther Onkol 179: 641-649, 2003.

20. Sakurada ATT, Kwee TC, Yamashita T, Nasu S, Horie T, van Cauteren $M$ and Imai $Y$ : Diagnostic performance of diffusion-weighted magnetic resonance imaging in esophageal cancer. Eur Radiol 19: 1461-1469, 2009.

21. Gideon P, Sorensen PS, Thomsen C, Stahlberg F, Gjerris F and Henriksen $\mathrm{O}$ : Increased brain water self-diffusion in patients with idiopathic intracranial hypertension. AJNR Am J Neuroradiol 16: 381-387, 1995.

22. Szafer A, Zhong J and Gore JC: Theoretical model for water diffusion in tissues. Magn Reson Med 33: 697-712, 1995.

23. Duong TQ, Ackerman JJ, Ying HS and Neil JJ: Evaluation of extra- and intracellular apparent diffusion in normal and globally ischemic rat brain via 19F NMR. Magn Reson Med 40: 1-13, 1998.

24. Haberkorn U, Strauss LG, Reisser C, et al: Glucose uptake, perfusion, and cell proliferation in head and neck tumors: relation of positron emission tomography to flow cytometry. J Nucl Med 32: 1548-1555, 1991.

25. Jacob R, Welkoborsky HJ, Mann WJ, Jauch M and Amedee R: [Fluorine-18]fluorodeoxyglucose positron emission tomography, DNA ploidy and growth fraction in squamous-cell carcinomas of the head and neck. ORL J Otorhinolaryngol Relat Spec 63: 307-313, 2001

26. Kitagawa Y, Sano K, Nishizawa S, et al: FDG-PET for prediction of tumour aggressiveness and response to intra-arterial chemotherapy and radiotherapy in head and neck cancer. Eur J Nucl Med Mol Imaging 30: 63-71, 2003.

27. Minn H, Joensuu H, Ahonen A and Klemi P: Fluorodeoxyglucose imaging: a method to assess the proliferative activity of human cancer in vivo. Comparison with DNA flow cytometry in head and neck tumors. Cancer 61: 1776-1781, 1988.

28. Shimada H, Nabeya Y, Okazumi S, et al: Prediction of survival with squamous cell carcinoma antigen in patients with resectable esophageal squamous cell carcinoma. Surgery 133: 486-494, 2003. 